\title{
A novel donor site mutation in LMNA gene leading to severe form of Dilated Cardiomyopathy in a proband of a family from Bihar, India
}

\author{
Soumi Das ${ }^{1 *}$, Amitabh Biswas', Mitali Kapoor', Sandeep Seth², Balram Bhargava², Vadlamudi Rao \\ From International Conference on Human Genetics and 39th Annual Meeting of the Indian Society of \\ Human Genetics (ISHG) \\ Ahmadabad, India. 23-25 January 2013
}

\section{Background}

Dilated Cardiomyopathy (DCM) is poorly understood in terms of their mechanistic pathways. It may lead to sudden cardiac death with a prevalence rate of 0.04-0.2\%. The cause of DCM is still unknown and referred to as Idiopathic Dilated Cardiomyopathy. There are many candidate genes associated with the DCM but most of the mutations are found in the LMNA and MYH7 genes.

\section{Methods}

The proband underwent Echocardiography and ECG to confirm the diagnosis. $5 \mathrm{ml}$ blood was collected and DNA was extracted using Phenol-chloroform method. The hot spot regions exon 23 of MYH7 gene, exon3 and exon 4 along with the intron3 of LMNA gene were sequenced using Sanger sequencing (ABI 3730xl). ACE $287 \mathrm{bpI} / \mathrm{D}$ and TNNT25bpI/D polymorphisms were also genotyped. In silico analysis of this novel mutation by using softwares, Human splicing finder (HSF) and MaxENT to understand the effect of mutation on splicing. The study was ethically approved by Institutional committee and informed written consent was taken from all participants.

\section{Results}

The proband aged 50yrs diagnosed with DCM, age of onset 45yrs, showing severe symptoms such as dyspnea, palpitation, fatigue and pedal edema under NYHA III classification showing dilated LV with EF 30\%. Proband's mother died due to heart problem but was not clinically confirmed for DCM and his sister had a suspicious death. The son of the proband aged 24 years has the same LMNA mutation but is asymptomatic presently.

A Novel donor splice site mutation $\mathrm{G}>\mathrm{C}$ transversion in intron3 of LMNA gene and a synonymous mutation $(\mathrm{C}>\mathrm{T}$ at codon 923) was found in MYH7 gene in proband. ACE and TNNT2 polymorphisms showed a heterozygous (ID) and homozygous (II) genotypes respectively. In silico analysis by HSF and MaxENT shows that the elimination of natural donor splice site leading to the use of a cryptic donor site which is located 7 nucleotide upstream of native splice site.

\section{Conclusion}

As reported in previous studies, LMNA gene mutations are associated to the severe form of DCM. From the above results, it may be concluded that the severe form of the disease is due to the splice site mutation.

\section{Authors' details}

'Department of Anthropology, University of Delhi, Delhi, India. ${ }^{2}$ Department of Cardiology, AllMS, India.

Published: 21 January 2014

doi:10.1186/1755-8166-7-S1-P35

Cite this article as: Das et al:: A novel donor site mutation in LMNA gene leading to severe form of Dilated Cardiomyopathy in a proband of a family from Bihar, India. Molecular Cytogenetics 2014 7(Suppl 1):P35. 